\title{
Resonance Fluorescence of GaAs Quantum Dots with Near-Unity Photon Indistinguishability
}

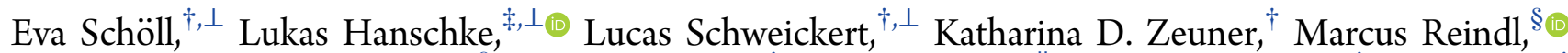
Saimon Filipe Covre da Silva, ${ }^{\S}$ Thomas Lettner, ${ }^{\dagger}$ Rinaldo Trotta, ${ }^{\dagger}$ Jonathan J. Finley, ${ }^{\ddagger}$ Kai Müller, ${ }^{\ddagger}$ Armando Rastelli, ${ }^{\S}$ Val Zwiller, ${ }^{\dagger}$ and Klaus D. Jöns ${ }^{*}, \dagger$

${ }^{\dagger}$ Department of Applied Physics, Royal Institute of Technology, Albanova University Centre, Roslagstullsbacken 21, 10691 Stockholm, Sweden

${ }^{\ddagger}$ Walter Schottky Institut and Physik Department, Technische Universität München, 85748 Garching, Germany

${ }^{\S}$ Institute of Semiconductor and Solid State Physics, Johannes Kepler University Linz, 4040 Linz, Austria

"Dipartimento di Fisica, Sapienza Università di Roma, Piazzale A. Moro 1, I-00185 Roma, Italy

\section{Supporting Information}

ABSTRACT: Photonic quantum technologies call for scalable quantum light sources that can be integrated, while providing the end user with single and entangled photons on demand. One promising candidate is strain free GaAs/AlGaAs quantum dots obtained by aluminum droplet etching. Such quantum dots exhibit ultra low multi-photon probability and an unprecedented degree of photon pair entanglement. However, different to commonly studied InGaAs/GaAs quantum dots obtained by the Stranski-Krastanow mode, photons with a near-unity indistinguishability from these quantum emitters have proven to be elusive so far. Here, we show on-demand generation of near-unity indistinguishable photons from these quantum emitters by exploring pulsed resonance fluorescence. Given the short intrinsic lifetime of excitons and trions confined in the GaAs quantum dots, we show single photon indistinguishability with a raw visibility of $V_{\text {raw }}=\left(95.0_{-6.1}^{+5.0}\right) \%$, without the need for Purcell enhancement. Our results represent a milestone in the advance of GaAs quantum dots by demonstrating the final missing property standing in the way of using these emitters as a key component in quantum communication applications, e.g., as quantum light sources for quantum repeater architectures.

KEYWORDS: Semiconductor quantum dot, resonance fluorescence, indistinguishable photons, Al droplet etching
$\mathrm{M}$ ost applications in photonic quantum technologies rely on clean quantum interference of deterministically generated single and entangled photons. Quantum indistinguishability is a crucial ingredient for the creation of higher NO0N states, ${ }^{1,2}$ quantum teleportation ${ }^{3}$ and swapping operations, ${ }^{4}$ boson-sampling, ${ }^{5,6}$ and photon-based quantum simulations. ${ }^{7}$ An ideal quantum light source thus needs to emit photons on demand, with high purity and near-unity indistinguishability as well as being scalable and interconnected with different quantum systems. Semiconductor quantum dots are proving to be the best sources that fulfill these requirements, ${ }^{8}$ delivering ultra bright sources of on-demand single photons at high rates compatible with photonic circuitry. Recently, GaAs quantum dots obtained by the infilling of nanoholes produced by local droplet etching ${ }^{9}$ have emerged to be one of the most promising deterministic solid-state quantum light sources, reporting the lowest multi-photon probability ${ }^{10}$ and the highest degree of polarization entanglement ${ }^{11}$ while also being the brightest entangled photon pair source reported. ${ }^{12}$ Entangled photons from these quantum dots have also been used to implement quantum tele- portation $^{13}$ and entanglement swapping protocols, ${ }^{14}$ thus proving their potential for quantum network applications. Furthermore, their short intrinsic lifetime enables highrepetition rate single-photon sources and, together with the high symmetry of the quantum dots, results in improved entanglement fidelities. ${ }^{11}$ Another advantage of these quantum dots is that they have reduced strain gradients compared to commonly studied dots obtained by the Stranski-Krastanow growth mode and are expected to have long nuclei ensemble spin coherence, promising for quantum-dot-based quantum memories. ${ }^{15}$ Additionally, their emission wavelength range makes them suitable for hybrid quantum photonic technologies since they can be tuned into resonance with quantum memories, e.g. rubidium atoms. ${ }^{16,17}$ However, near-unity indistinguishable photons, a crucial element for photonic quantum technologies, was missing from this type of quantum

Received: December 24, 2018

Revised: $\quad$ March 11, 2019

Published: March 13, 2019 


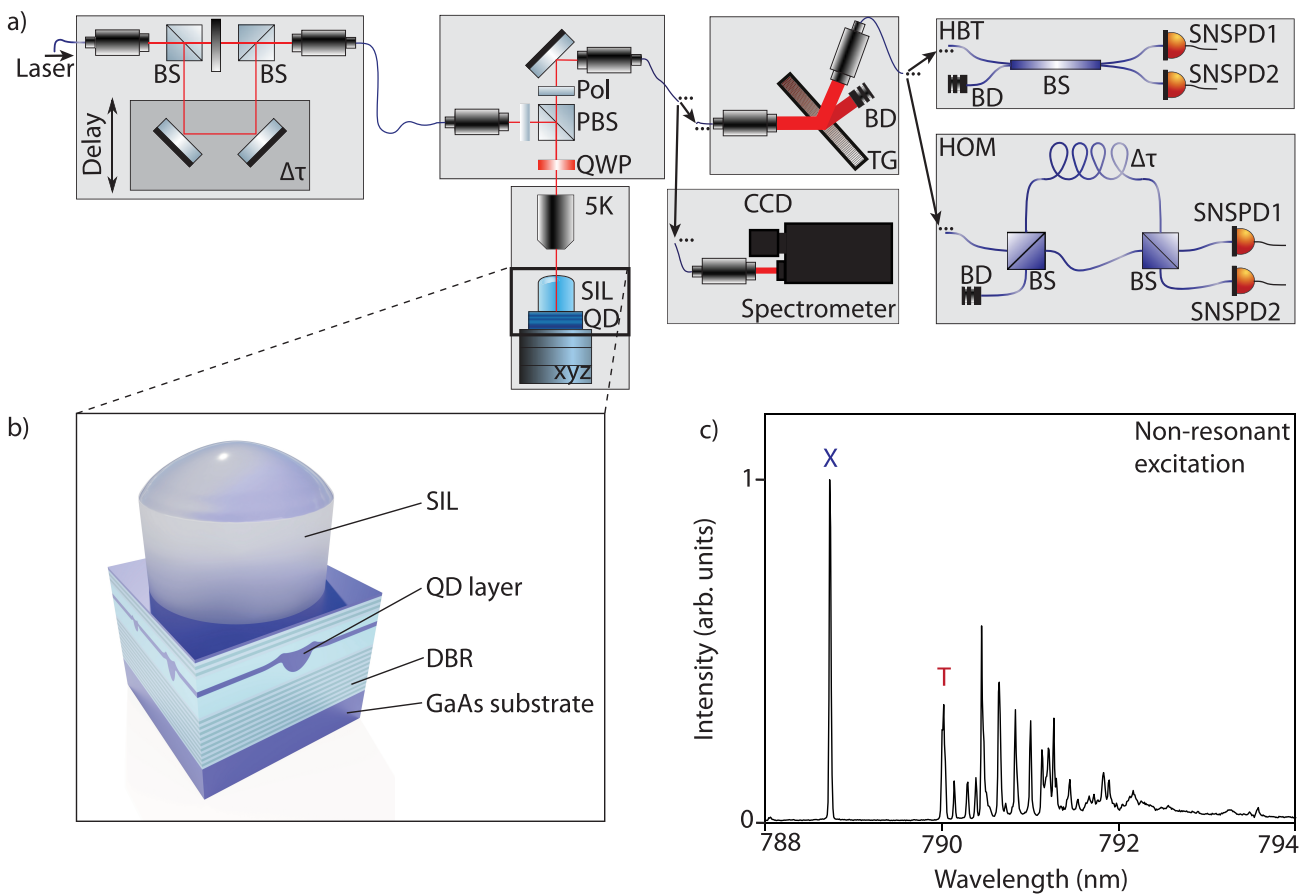

Figure 1. (a) The modular setup consisting of laser excitation with delay line, the confocal microscopy setup with polarization suppression, the transmission spectrometer, the Hanbury-Brown and Twiss setup (HBT), and the Hong-Ou-Mandel setup (HOM). BS, beam splitter; BD, beam dump; TG, transmission grating; SNSPD, superconducting nanowire single photon detector; Pol, polarizer; PBS, polarizing beam splitter; QWP, quarter waveplate; SIL, solid immersion lens. (b) Schematic illustration of the sample structure. (c) Spectrum of QD1 under non-resonant excitation.

dots until now. Strong charge fluctuations at the vicinity of the quantum dot, ${ }^{18,19}$ induced by the droplet growth method ${ }^{20}$ and faster phonon-induced pure dephasing ${ }^{21}$ and zero-phonon broadening $^{22}$ were suspected to be the main causes of the lower quantum interference visibility for these quantum emitters. In this letter, we apply cross-polarized pulsed resonance fluorescence to show that quantum dots derived from droplet etching do not suffer from additional dephasing mechanisms at short time scales and exhibit near-unity indistinguishability of on-demand generated single photons. Remarkably, high quantum interference visibility values of $V_{\text {raw }}=\left(95.0_{-6.1}^{+5.0}\right) \%$ are achieved without the need of Purcell enhancement using microcavities. ${ }^{23-25}$ Instead, we capitalize on the intrinsically short lifetime of the excited states of these quantum emitters. ${ }^{26}$

For pulsed resonant s-shell excitation, ${ }^{27}$ a polarization suppression setup was constructed similar to that in ref 28 but with the second polarizing beam splitter (PBS) replaced by a nanoparticle linear film polarizer, as illustrated in Figure 1 a. The sample was mounted in a low vibration closed-cycle cryostat and cooled to $5 \mathrm{~K}$. For excitation, a tunable, linearly polarized laser was used with a repetition rate of $80 \mathrm{MHz}$ and pulse duration of 5.0 ps after sending the pulse through a pulse slicer. The excitation beam was directed onto the sample via the polarizing beam splitter, through an objective with $N A=0.81$, and focused onto the quantum dot of interest using a solid immersion lens (SIL), directly attached to the sample surface. The signal was collected through the same optics in a confocal geometry and separated from the backscattered excitation laser by the polarizing beam splitter and a linear polarizer oriented perpendicular to the laser polarization. Further improvement of the laser suppression was achieved by spatial filtering since a small portion of the backscattered laser has a component perpendicular to the original polarization with a four-leaf clover patterned beam profile. $^{29}$

To perform photoluminescence measurements, the signal was coupled through a spectrometer onto a silicon CCD. For correlation measurements, the resonance fluorescence signal was further filtered with a home-built transmission spectrometer having a bandwidth of $19 \mathrm{GHz}$ and an end-to-end efficiency exceeding $60 \%$. Second-order intensity correlation measurements were carried out with a Hanbury-Brown and Twiss type setup realized with a fiber coupled 50:50 beam splitter connected to two superconducting nanowire single photon detectors (SNSPD) with efficiencies of $50 \%$ and $60 \%$, a timing jitter of 20 and $30 \mathrm{ps,} \mathrm{and} \mathrm{dark} \mathrm{count} \mathrm{rates} \mathrm{of} 0.006$ and $0.017 \mathrm{dcts} / \mathrm{s}$. The detection events are recorded in a timetag file along with laser excitation events and analyzed with our Extensible Timetag Analyzer (ETA) software. ${ }^{30}$ To determine the indistinguishability of two consecutively emitted photons, two-photon interference was measured in a Hong-OuMandel (HOM) type experiment. In order to interfere, these photons must impinge on a beam splitter with excellent spatial and temporal overlap. The temporal overlap is achieved by sending the signal into an unbalanced fiber-based MachZehnder interferometer with a path-length difference of 2 ns. The two output ports of the Mach-Zehnder interferometer are connected to a SNSPD each. Depending on the paths the photons take, they can arrive on the beam splitter simultaneously or with a time delay of 2 or $4 \mathrm{~ns}$, resulting in the characteristic quintuplet for Hong-Ou-Mandel measurements ${ }^{31}$ in the histogram. The temporal overlap of the photons on the second beam splitter is ensured by splitting the excitation pulse into two identical pulses using another unbalanced Mach-Zehnder interferometer with variable 

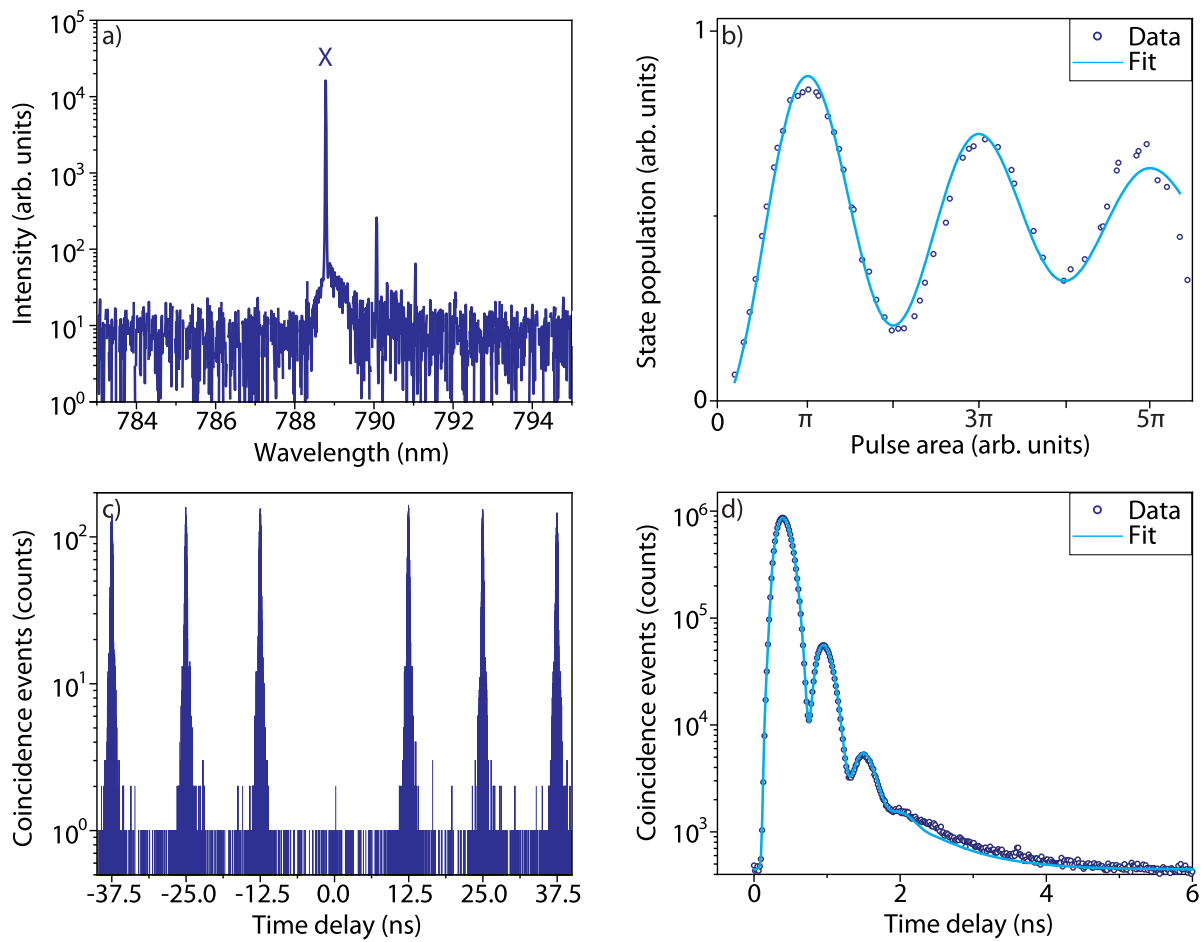

Figure 2. Characterization of the neutral exciton under pulsed resonant s-shell excitation. (a) Resonance fluorescence spectrum in a semilogarithmic plot. (b) Excitation laser power-dependent Rabi oscillation up to a pulse area of $5 \pi$. From our fit we extract an occupation probability of $85 \%$ under $\pi$-pulse excitation. (c) Second-order intensity correlation histogram yielding $g^{(2)}(0)=(2.5 \pm 0.2) \times 10^{-3}$ (d) Semi-logarithmic plot of the lifetime measurement with oscillations due to the fine structure splitting. The fit gives a lifetime of $196 \pm 2$ ps and a fine structure splitting of $7.44 \pm 0.05 \mu \mathrm{eV}$.

delay. This delay is precisely tuned to the fixed fiber delay in the Hong-Ou-Mandel setup by measuring the interference of overlapping short laser pulses using the same detectors as for all the correlation measurements. For the best possible time resolution, unsliced laser pulses with a pulse duration of 1.94 ps were used.

The GaAs quantum dot sample investigated in this work was grown by molecular beam epitaxy using the aluminum droplet etching technique. The quantum dot layer was embedded in a $\lambda$-cavity made of $\mathrm{Al}_{0.4} \mathrm{Ga}_{0.6} \mathrm{As}(123 \mathrm{~nm})$ with 9.5 pairs of bottom and 2.5 pairs of top distributed Bragg reflectors (DBR) consisting of $\mathrm{Al}_{0.95} \mathrm{Ga}_{0.05} \mathrm{As} / \mathrm{Al}_{0.2} \mathrm{Ga}_{0.8} \mathrm{As} \lambda / 4$-thick layers, as depicted in Figure 1 b. The structure was finished by a $4 \mathrm{~nm}$ thick protective GaAs layer. The Al-droplet etching method allows the growth of highly symmetric quantum dots with a low fine structure splitting (FSS). ${ }^{32}$ This sample structure shows an extraction efficiency of $20.0 \pm 3.2 \%$ into the first lens for a quantum dot well-positioned under the SIL.

In Figure $1 \mathrm{c}$ we show the spectrum of QD1 subject to pulsed non-resonant excitation at a wavelength of $781 \mathrm{~nm}$. The neutral exciton (X), emitting at $788.73 \mathrm{~nm}$, is isolated from the rest of emission lines at longer wavelengths, which are attributed to electron-hole recombination in the presence of additional carriers in the quantum dot which stem from nearby confined states due to the weak confinement in shallow quantum dots. ${ }^{33}$ The trion $(\mathrm{T})$ transition corresponds to the peak at $790.02 \mathrm{~nm}$. To resonantly excite an s-shell transition ( $\mathrm{X}$ or $\mathrm{T}$ ), we tune the energy of the excitation laser to the transition energy, ideally resulting in photoluminescence only from this specific transition. Furthermore, the dephasing due to interactions with phonons and nearby trapped charge carriers is reduced. ${ }^{34}$ To address the electric environment of the dot, we additionally illuminate the sample with a low intensity white light source. ${ }^{35,36}$ This results in a very clean spectrum with only one prominent line of the exciton transition and minor contribution of less than $2 \%$ from the trion transition as shown in a semi-logarithmic plot in Figure 2 a.

To show that this excitation scheme addresses the quantum dot coherently, we performed power-dependent pulsed resonance fluorescence measurements. In Figure $2 \mathrm{~b}$, Rabi oscillations of the integrated intensity of the neutral exciton transition as a function of the excitation pulse area are shown. By exciting the quantum dot with a power corresponding to a pulse area of $\pi$, the population of the two-level system of the quantum dot is maximally inverted, preparing the quantum dot in the excited state. The procedure used to fit the data is explained in the Supporting Information. We extract a population probability for the neutral exciton state of $85 \%$ under $\pi$-pulse excitation. For all further measurements, the quantum dot is excited with a $\pi$-pulse.

The second-order intensity correlation function shown in Figure 2 c shows nearly background free single photon emission. By calculating the ratio of the area of the center peak and average area of eight side peaks in a time window of $3.2 \mathrm{~ns}$ each, a measured degree of second-order coherence of $g^{(2)}(0)=(2.5 \pm 0.2) \times 10^{-3}$ is determined. We attribute the deviation from 0 solely to re-excitation ${ }^{37}$ and conclude that there is negligible residual excitation laser present in the correlation measurement.

Figure $2 \mathrm{~d}$ shows the lifetime measurement of the resonantly excited neutral exciton in a semi-logarithmic plot. The exponential decay is observed with a periodic modulation. ${ }^{38}$ 

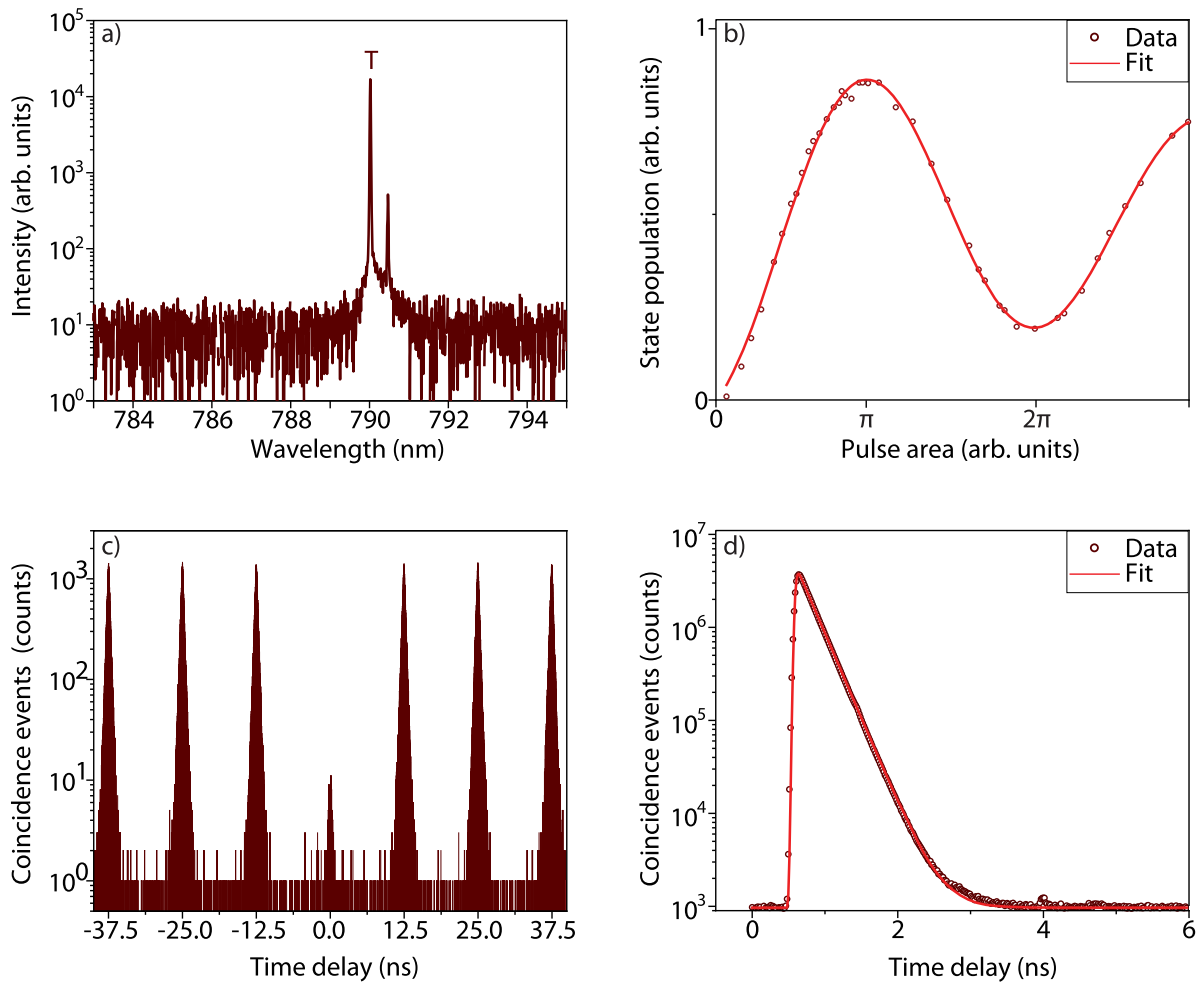

Figure 3. Characterization of the trion under pulsed s-shell resonant excitation. (a) Resonance fluorescence spectrum in a semi-logarithmic plot. The origin of the $\approx 30 \times$ less intense line is discussed in the main text. (b) Rabi oscillation up to a pulse area of $3 \pi$. (c) Second-order intensity correlation histogram yielding $g^{(2)}(0)=(6.7 \pm 0.4) \times 10^{-3}$ (d) Semi-logarithmic plot of the lifetime measurement fitted with a single exponential decay giving us a lifetime of $236 \pm 2$ ps.

Due to the exchange interaction between the electron and hole spins, the degeneracy of the exciton states of the quantum dot is lifted, leading to two linearly cross-polarized fine structure (FS) states with an energy splitting of $E_{\mathrm{Fss}}$. During excitation, the spin of the exciton state is determined by the polarization of the excitation pulse. ${ }^{39,40}$ Then, the spin starts precessing on the equator of the Bloch sphere, oscillating between the two orthogonal fine structure states. The polarization of the emitted photon is set by the spin at the time of the recombination. Since one polarization component is suppressed by the polarizers in our setup, the intensity of the detected signal oscillates with a frequency $\nu=\frac{E_{\mathrm{FSS}}}{h} .{ }^{41}$ The data is modeled with a fit explained in the Supporting Information, which yields a lifetime of $196 \pm 2$ ps and a fine structure splitting of $7.44 \pm 0.05 \mu \mathrm{eV}$.

In order to point out similarities with and differences to the neutral exciton, we investigate the resonantly excited charged exciton as well. Figure 3 a shows the spectrum of the trion of QD1 under pulsed resonant excitation. We observe an additional line close to the trion transition with $\approx 30$ times lower intensity, which might be a higher charge state emitting after a second capture process. Similar to the neutral exciton, we observe clear Rabi oscillations as a function of the excitation pulse area, as shown in Figure $3 \mathrm{~b}$, and a maximum population inversion probability of $86 \%$ under $\pi$-pulse excitation. The second-order intensity correlation function yields a measured degree of second-order coherence of $(6.7 \pm 0.4) \times 10^{-3}$, as shown in Figure $3 c$, confirming that the laser suppression is very good. In Figure $3 \mathrm{~d}$ we show a semi-logarithmic plot of a lifetime measurement. A single exponential fit gives a lifetime of $236 \pm 2$ ps. As opposed to the lifetime measurement of the neutral exciton in Figure $3 \mathrm{~d}$, this measurement shows no quantum beats, due to the lack of fine structure splitting of the trion state. ${ }^{42}$

Having confirmed low multi-photon emission probability for both neutral and charged excitons under $\pi$-pulse resonant excitation, we continue to investigate the indistinguishability of consecutively emitted photons using a two-photon interference measurement, as described above. In Figure $4 \mathrm{a}$ and $\mathrm{b}$ the center peak quintuplet for two-photon interference measurements of the neutral exciton and trion of QD1 are shown. The relative peak heights originate from different combinations of long and short paths in the Mach-Zehnder interferometer two consecutive photons can take. In the Hong-Ou-Mandel measurement of the neutral exciton, the same oscillations as in the lifetime measurements are visible.

In the limit of $g^{(2)}(0)=0$, the visibility of two-photon interference can be calculated from the area of the three center peaks $A_{1,2,3}$ by $V=1-\frac{2 \times A_{2}}{A_{1}+A_{3}}$, where $V=100 \%$ corresponds to perfectly indistinguishable photons. ${ }^{31}$ To calculate the peak area of the three center peaks, the coincidence events are summed up in time windows which are individual for every transition (see details in the Supporting Information). The uncorrected visibilities for QD1 are $V_{\text {raw }}=94.9 \%$ with a statistical error $+5.1 \%$ and $-6.4 \%$ for the neutral exciton and $V_{\text {raw }}=(88.5 \pm 3.3) \%$ for the trion. To compensate for imperfections in the setup, we measure the classical interference fringe visibility with a narrow continuous wave diode laser to be $(97.5 \pm 0.1) \%$, which yields the upper measurable bound of the visibility in this setup. In general, we 

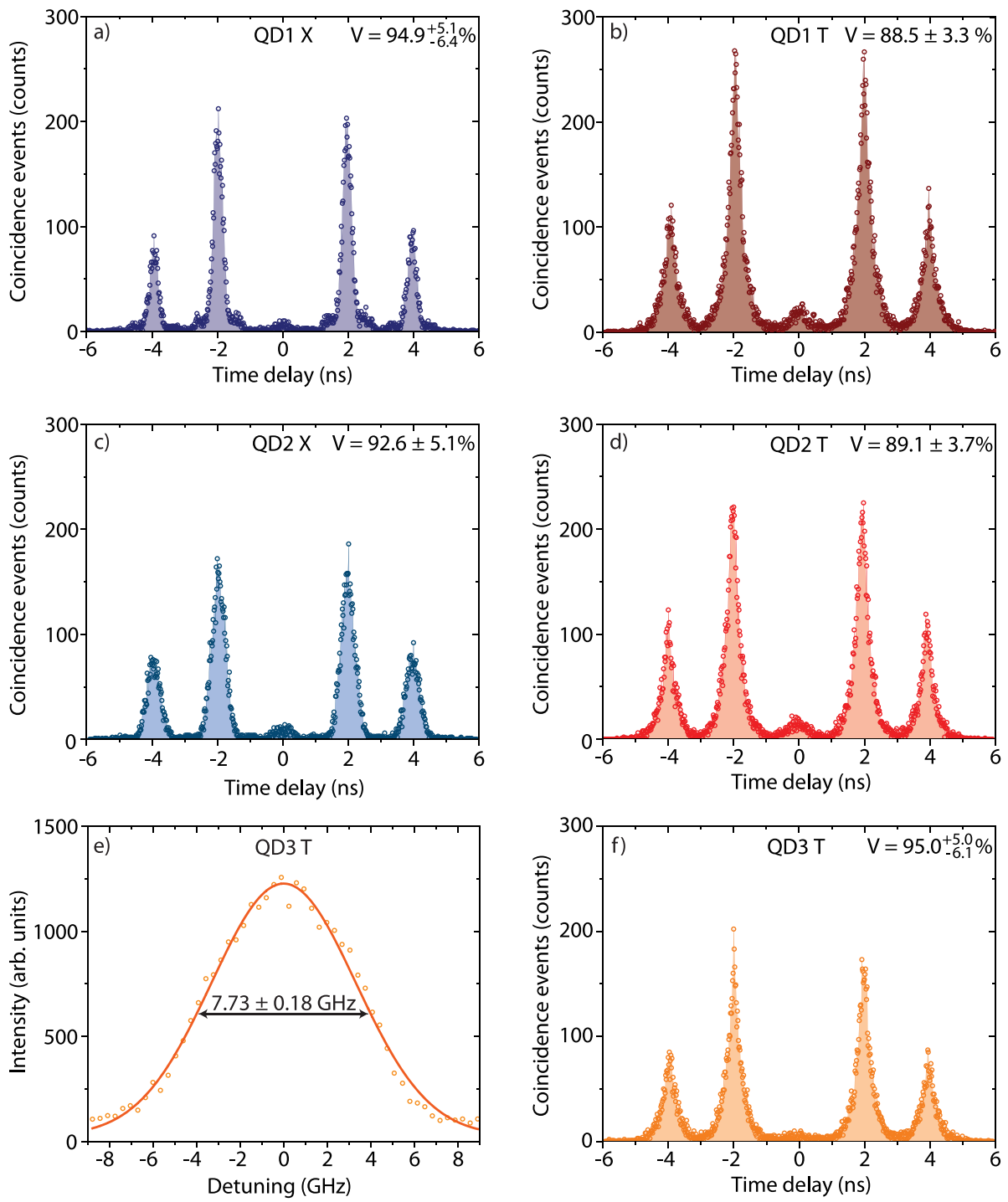

Figure 4. Hong-Ou-Mandel measurements under resonant s-shell excitation for the (a) neutral exciton of QD1 $\left(V=94.9_{-6.4}^{+5.1} \%\right)$, (b) trion of QD1 $(V=88.5 \pm 3.3 \%)$, (c) neutral exciton of QD2 $(V=92.6 \pm 5.1 \%)$, (d) trion of QD2 $(V=89.1 \pm 3.7 \%)$, and (f) trion of QD3 $\left(V=95.0_{-6.1}^{+5.0} \%\right)$. The visibilities are calculated by summing up the peak areas of the three center peaks. The method is explained in more detail in the Supporting Information; (e) line width of the trion of QD3 with a Gaussian fit $(\Delta \nu=7.73 \pm 0.18 \mathrm{GHz})$.

are able to show very high indistinguishability visibilities by performing the Hong-Ou-Mandel measurement on further dots, as shown in Figure $4 \mathrm{c}$, d, and $\mathrm{f}$. Here we obtain a visibility of $V_{\text {raw }}=(92.6 \pm 5.1) \%$ for the neutral exciton, $V_{\text {raw }}=(89.1 \pm 3.7) \%$ for the trion of QD2, and $V_{\text {raw }}=\left(95.0_{-6.1}^{+5.0}\right) \%$ for the trion of QD3. This value is the highest obtained visibility for on-demand sources without Purcell enhancement or more elaborate excitation techniques. $^{43}$ We would like to note that fitting the Hong-OuMandel data, instead of summing up the data in specified time windows, very often overestimates the two-photon interference visibility, in particular if the data were collected with low timing resolution. Especially when the low time resolution is masking quantum beats and the dip at zero time delay, fitting can wrongly increase the visibility and even lead to unphysical results, i.e., visibilities above $100 \%$. This result is even independent of the used fit function (see details in the Supporting Information). We measure the line width of the trion transition of QD3 by slowly scanning a Fabry-Pérot interferometer with a resolution of $28 \mathrm{MHz}$ over the line and recording the signal on a SNSPD. Fitting the data with a Gaussian peak provides a line width of $7.73 \pm 0.18 \mathrm{GHz}$ and is shown in Figure 4 e. Considering a lifetime of 228 ps, we show that the line width is a factor of 10 larger than the Fourier limit. As we are still measuring a very high $\mathrm{HOM}$ visibility for this transition, this indicates negligible spectral wandering on short time scales.

We point out that our two-photon interference visibility value of $V=\left(95.0_{-6.1}^{+5.0}\right) \%$ is the highest raw value measured for any on-demand source without a micro-cavity and marks an important milestone for quantum dots derived from droplet etching. Near-unity indistinguishability was the last missing quantum optical property to put GaAs quantum dots on the horizon for future quantum communication and quantum information processing applications. On the basis of our results, we foresee that photonic structures other than cavities, e.g., waveguides, trumpets, and nanowires ${ }^{44,45}$ to enhance light extraction efficiency from solid-state emitters, can be used to 
achieve even higher levels of indistinguishability without the need of Purcell enhancement.

\section{ASSOCIATED CONTENT}

\section{S Supporting Information}

The Supporting Information is available free of charge on the ACS Publications website at DOI: 10.1021/acs.nanolett.8b05132.

Resonance fluorescence of GaAs quantum dots with near-unity photon indistinguishability; Rabi oscillation; time-correlated single-photon counting fit function including convolution with internal response function; polarization-resolved photoluminescence spectroscopy; and analysis of two-photon interference measurements (PDF)

\section{AUTHOR INFORMATION}

\section{Corresponding Author}

*E-mail: klausj@kth.se.

\section{ORCID}

Lukas Hanschke: 0000-0002-1943-7603

Marcus Reindl: 0000-0001-7333-515X

Klaus D. Jöns: 0000-0002-5814-7510

\section{Author Contributions}

${ }^{\perp}$ E. Schöll, L. Hanschke, and L. Schweickert contributed equally to this work. E.S. and K.D.J. built the setup with the help from K.D.Z., L.S., and T.L., E.S., L.H., L.S., K.D.Z., and K.D.J. performed the measurements. E.S., L.H., L.S., and K.D.Z. carried out the data analysis with help from K.D.J. S.F.C.S. and A.R. designed, optimized, and grew the quantum dot sample. M.R. and R.T. characterized the quantum dot sample, helping to optimize the sample. T.L. fabricated the final device with the help from R.T. E.S. and K.D.J. wrote the manuscript with help from all of the authors. K.D.J. conceived the experiment and supervised the project.

\section{Notes}

The authors declare no competing financial interest.

\section{ACKNOWLEDGMENTS}

This project has received funding from the European Union's Horizon 2020 Research and Innovation Program (820423; S2QUIP), the European Research Council (ERC) under the European Union's Horizon 2020 Research and Innovation Programme (SPQRel; 679183), the FWF (P 29603, P 30459), the Linz Institute of Technology, the German Federal Ministry of Education and Research via the funding program Photonics Research Germany (13N14846), Q.Com (16KIS0110) and Q.Link.X, the DFG via the Nanosystem Initiative Munich, the MCQST, the Knut and Alice Wallenberg Foundation grant "Quantum Sensors", the Swedish Research Council (VR) through the VR grant for international recruitment of leading researchers (ref. 2013-7152), and the Linnæus Excellence Center ADOPT. K.D.Z. gratefully acknowledges funding by the Dr. Isolde Dietrich Foundation. K.M. acknowledges support from the Bavarian Academy of Sciences and Humanities. K.D.J. acknowledges funding from the Swedish Research Council (VR) via the starting grant HyQRep (ref. 2018-04812). A.R. acknowledges fruitful discussions with Y. Huo, G. Weihs, R. Keil, and S. Portalupi.

\section{REFERENCES}

(1) Walther, P.; Pan, J.-W.; Aspelmeyer, M.; Ursin, R.; Gasparoni, S.; Zeilinger, A. Nature 2004, 429, 158.

(2) Nagata, T.; Okamoto, R.; O’Brien, J. L.; Sasaki, K.; Takeuchi, S. Science 2007, 316, 726-729.

(3) Bouwmeester, D.; Pan, J.-W.; Mattle, K.; Eibl, M.; Weinfurter, H.; Zeilinger, A. Nature 1997, 390, 575-579.

(4) Pan, J.-W.; Bouwmeester, D.; Weinfurter, H.; Zeilinger, A. Phys. Rev. Lett. 1998, 80, 3891-3894.

(5) Spring, J. B.; Metcalf, B. J.; Humphreys, P. C.; Kolthammer, W. S.; Jin, X.-M.; Barbieri, M.; Datta, A.; Thomas-Peter, N.; Langford, N. K.; Kundys, D.; Gates, J. C.; Smith, B. J.; Smith, P. G. R.; Walmsley, I. A. Science 2013, 339, 798-801.

(6) Broome, M. A.; Fedrizzi, A.; Rahimi-Keshari, S.; Dove, J.; Aaronson, S.; Ralph, T. C.; White, A. G. Science 2013, 339, 794-798.

(7) Aspuru-Guzik, A.; Walther, P. Nat. Phys. 2012, 8, 285.

(8) Senellart, P.; Solomon, G.; White, A. Nat. Nanotechnol. 2017, 12, 1026-1039.

(9) Huber, D.; Reindl, M.; Huo, Y.; Huang, H.; Wildmann, J. S.; Schmidt, O. G.; Rastelli, A.; Trotta, R. Nat. Commun. 2017, 8, 15506.

(10) Schweickert, L.; Jöns, K. D.; Zeuner, K. D.; Covre da Silva, S. F.; Huang, H.; Lettner, T.; Reindl, M.; Zichi, J.; Trotta, R.; Rastelli, A.; Zwiller, V. Appl. Phys. Lett. 2018, 112, 093106.

(11) Huber, D.; Reindl, M.; Covre da Silva, S. F.; Schimpf, C.; Martín-Sánchez, J.; Huang, H.; Piredda, G.; Edlinger, J.; Rastelli, A.; Trotta, R. Phys. Rev. Lett. 2018, 121, 033902.

(12) Chen, Y.; Zopf, M.; Keil, R.; Ding, F.; Schmidt, O. G. Nat. Commun. 2018, 9, 2994.

(13) Reindl, M.; Huber, D.; Schimpf, C.; da Silva, S. F. C.; Rota, M. B.; Huang, H.; Zwiller, V.; Jöns, K. D.; Rastelli, A.; Trotta, R. Sci. Adv. 2018, 4, eaau1255.

(14) Basso Basset, F.; Rota, M. B.; Schimpf, C.; Tedeschi, D.; Zeuner, K. D.; Covre da Silva, S. F.; Reindl, M.; Zwiller, V.; Jöns, K. D.; Rastelli, A.; Trotta, R. Entanglement swapping with photons generated on-demand by a quantum dot. 2019, arXiv:1901.06646.arXiv.org e-Prints. https://arxiv.org/abs/1901.06646

(15) Gangloff, D. A.; Éthier-Majcher, G.; Lang, C.; Denning, E. V.; Bodey, J. H.; Jackson, D. M.; Clarke, E.; Hugues, M.; Le Gall, C.; Atatüre, M. Science 2019, eaaw2906.

(16) Akopian, N.; Wang, L.; Rastelli, A.; Schmidt, O.; Zwiller, V. Nat. Photonics 2011, 5, 230-233.

(17) Schweickert, L.; Jöns, K. D.; Namazi, M.; Cui, G.; Lettner, T.; Zeuner, K. D.; Scavuzzo Montaña, L.; Covre da Silva, S. F.; Reindl, M.; Huang, H.; Trotta, R.; Rastelli, A.; Zwiller, V.; Figueroa, E. Electromagnetically Induced Transparency of On-demand Single Photons in a Hybrid Quantum Network. 2018, arXiv:1808.05921.arXiv.org e-Print archive. https://arxiv.org/abs/1808.05921.

(18) Heyn, C.; Zocher, M.; Pudewill, L.; Runge, H.; Küster, A.; Hansen, W. J. Appl. Phys. 2017, 121, 044306.

(19) Liu, J.; Konthasinghe, K.; Davanço, M.; Lawall, J.; Anant, V.; Verma, V.; Mirin, R.; Nam, S. W.; Song, J. D.; Ma, B.; Chen, Z. S.; Ni, H. Q.; Niu, Z. C.; Srinivasan, K. Phys. Rev. Appl. 2018, 9, 064019.

(20) Heyn, C.; Stemmann, A.; Köppen, T.; Strelow, C.; Kipp, T.; Grave, M.; Mendach, S.; Hansen, W. Appl. Phys. Lett. 2009, 94, 183113.

(21) Axt, V. M.; Kuhn, T.; Vagov, A.; Peeters, F. M. Phys. Rev. B: Condens. Matter Mater. Phys. 2005, 72, 125309.

(22) Tighineanu, P.; Dreeßen, C. L.; Flindt, C.; Lodahl, P.; Sørensen, A. S. Phys. Rev. Lett. 2018, 120, 257401.

(23) Somaschi, N.; et al. Nat. Photonics 2016, 10, 340-345.

(24) Ding, X.; He, Y.; Duan, Z.-C.; Gregersen, N.; Chen, M.-C.; Unsleber, S.; Maier, S.; Schneider, C.; Kamp, M.; Höfling, S.; Lu, C.Y.; Pan, J.-W. Phys. Rev. Lett. 2016, 116, 020401.

(25) Bennett, A. J.; Lee, J. P.; Ellis, D. J. P.; Meany, T.; Murray, E.; Floether, F. F.; Griffths, J. P.; Farrer, I.; Ritchie, D. A.; Shields, A. J. Sci. Adv. 2016, 2, No. e1501256.

(26) Kiraz, A.; Atatüre, M.; Imamoğlu, A. Phys. Rev. A: At., Mol., Opt. Phys. 2004, 69, 032305. 
(27) He, Y.-M.; He, Y.; Wei, Y.-J.; Wu, D.; Atatüre, M.; Schneider, C.; Höfling, S.; Kamp, M.; Lu, C.-Y.; Pan, J.-W. Nat. Nanotechnol. 2013, 8, 213-217.

(28) Kuhlmann, A. V.; Houel, J.; Brunner, D.; Ludwig, A.; Reuter, D.; Wieck, A. D.; Warburton, R. Rev. Sci. Instrum. 2013, 84, 073905. (29) Novotny, L.; Grober, R. D.; Karrai, K. Opt. Lett. 2001, 26, 789791.

(30) Schweickert, L.; Lin, Z.; Zeuner, K. D.; Lettner, T.; Gyger, S.; Zichi, J.; Schöll, E.; Jöns, K. D.; Zwiller, V. ETA is a graphical eventdriven programming language for time-tag processing. Zenodo: 2018; https://github.com/timetag;

(31) Santori, C.; Fattal, D.; Vucković, J.; Solomon, G. S.; Yamamoto, Y. Nature 2002, 419, 594-7.

(32) Huo, Y. H.; Rastelli, A.; Schmidt, O. G. Appl. Phys. Lett. 2013, $102,152105$.

(33) Musial, A.; Gold, P.; Andrzejewski, J.; Löffler, A.; Misiewicz, J.; Höfling, S.; Forchel, A.; Kamp, M.; Sęk, G.; Reitzenstein, S. Phys. Rev. B: Condens. Matter Mater. Phys. 2014, 90, 045430.

(34) Ates, S.; Ulrich, S. M.; Reitzenstein, S.; Löffler, A.; Forchel, A.; Michler, P. Phys. Rev. Lett. 2009, 103, 167402.

(35) Gazzano, O.; Michaelis de Vasconcellos, S.; Arnold, C.; Nowak, A.; Galopin, E.; Sagnes, I.; Lanco, L.; Lemaître, A.; Senellart, P. Nat. Commun. 2013, 4, 1425.

(36) Reindl, M.; Jöns, K. D.; Huber, D.; Schimpf, C.; Huo, Y.; Zwiller, V.; Rastelli, A.; Trotta, R. Nano Lett. 2017, 17, 4090-4095.

(37) Fischer, K. A.; Müller, K.; Lagoudakis, K. G.; Vučković, J. New J. Phys. 2016, 18, 113053.

(38) Dada, A. C.; Santana, T. S.; Koutroumanis, A.; Ma, Y.; Park, S.I.; Song, J.; Gerardot, B. D. Phys. Rev. B: Condens. Matter Mater. Phys. 2017, 96, 081404.

(39) Kodriano, Y.; Schwartz, I.; Poem, E.; Benny, Y.; Presman, R.; Truong, T. A.; Petroff, P. M.; Gershoni, D. Phys. Rev. B: Condens. Matter Mater. Phys. 2012, 85, 241304.

(40) Müller, K.; Kaldewey, T.; Ripszam, R.; Wildmann, J. S.; Bechtold, A.; Bichler, M.; Koblmüller, G.; Abstreiter, G.; Finley, J. Sci. Rep. 2013, 3, 1906.

(41) Flissikowski, T.; Hundt, A.; Lowisch, M.; Rabe, M.; Henneberger, F. Phys. Rev. Lett. 2001, 86, 3172-3175.

(42) Bayer, M.; Ortner, G.; Stern, O.; Kuther, A.; Gorbunov, A. A.; Forchel, A.; Hawrylak, P.; Fafard, S.; Hinzer, K.; Reinecke, T. L.; Walck, S. N.; Reithmaier, J. P.; Klopf, F.; Schäfer, F. Phys. Rev. B: Condens. Matter Mater. Phys. 2002, 65, 195315.

(43) Wei, Y.-J. J.; He, Y.-M. M.; Chen, M.-C. C.; Hu, Y.-N. N.; He, Y.-M. M.; Wu, D.; Schneider, C.; Kamp, M.; Höfling, S.; Lu, C.-Y. Y.; Pan, J.-W. W. Nano Lett. 2014, 14, 6515-6519.

(44) Gregersen, N.; McCutcheon, D. P. S.; Mørk, J.; Gérard, J.-M.; Claudon, J. Opt. Express 2016, 24, 20904-20924.

(45) Bulgarini, G.; Reimer, M. E.; Bouwes Bavinck, M.; Jöns, K. D.; Dalacu, D.; Poole, P. J.; Bakkers, E. P. A. M.; Zwiller, V. Nano Lett. 2014, 14, 4102-4106. 